\title{
A Computer Memory Based on Cold Atoms and Light
}

\author{
Merging ideas from neuroscience, machine learning, and quantum \\ technology, researchers propose a new information-storage device. \\ By Sophia Chen
}

M any recent computing advances derive their inspiration from models of the human brain. For example, researchers have created a machine-learning model that mimics the brain's ability to recognize new patterns by recalling previously encountered ones. So far, implementations of "associative memory" have largely involved conventional silicon-chip-based computers. Now, Benjamin Lev of Stanford University and colleagues propose a way of implementing associative memory with multiple Bose-Einstein condensates (BECs) and an optical cavity. The researchers say that their method should be better at learning and recognizing patterns than the standard associative memory design.

A computer with associative memory stores information in a mathematical function that looks like a potential energy landscape with many local minima. Each local minimum corresponds to a separate piece of information. To retrieve that information, the device is initialized in some state close to the relevant minimum, and it then finds that minimum. This process effectively reconstructs data from imprecise versions of

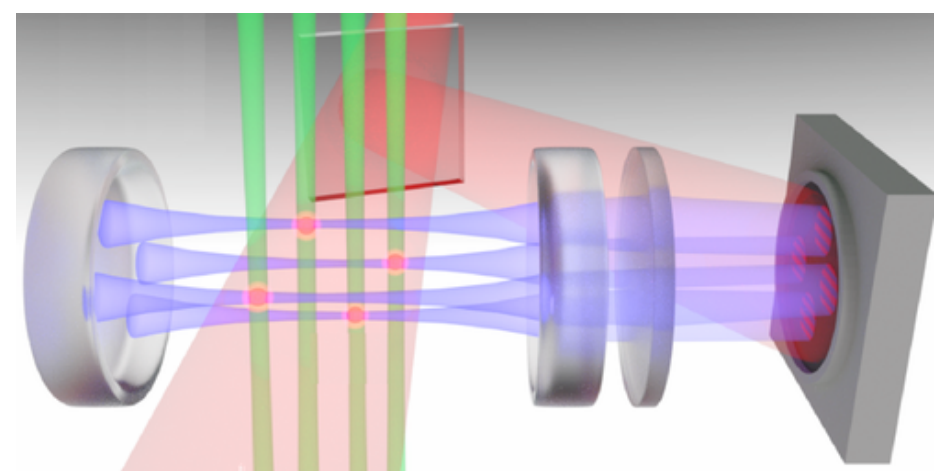

Credit: B. Marsh et al. [1] that data. While everyday technologies typically don't use associative memory techniques, researchers are interested in them because of their speed and their robustness to user mistakes.

The researchers' proposed device stores information in the energy landscape of multiple, separated BECs contained within the same optical cavity. The spin of each BEC interacts with that of the others by scattering photons in the cavity. They can engineer the energy landscape of the system by manipulating the position of each BEC. To retrieve information, the BECs are collectively initialized in a particular spin state, which relaxes into an energy minimum that is imaged using light emitted from the cavity. The researchers think they can build this device in the near term, as they have already demonstrated all the elements in the design.

Sophia Chen is a freelance science writer based in Columbus, Ohio.

\section{REFERENCES}

1. B. P. Marsh et al., "Enhancing associative memory recall and storage capacity using confocal cavity QED," Phys. Rev. X 11, 021048 (2021). 